\title{
COMPUTER CONTROLS OF THE CERN PS RADIO-FREQUENCY SYSTEM
}

\author{
G.P. BENINCASA and R. GAROBY \\ CERN/PS Division, 1211 Geneva 23, Switzerland
}

\begin{abstract}
The PS computer control project has recently been terminated with the integration of the radio-frequency system. While the general controls frame was used, special software and hardware solutions were also necessary to cope with the peculiarities of the rf system. After a brief description of both the rf process and the PS control system, this paper recalls the various operational requirements and real-time constraints and relates on the criteria and methods followed during the design phase. Some implementation is illustrated with emphasis on diagnostics and measurements.
\end{abstract}

\section{Introduction}

The recent years have seen at CERN a spectacular growth in the number of accelerators and in complexity of the operations. The PS has been the centre of this upheaval which transformed it from a simple-operation proton supplier to the heart of this "particle network" $[1,2]$.

To deal with this increased complexity of operation, a new computer system has been progressively installed to control the PS complex [3] during the last seven years. This system covers several processes: the PS accelerator itself, the PS Booster, the antiproton accumulator AA, the various beam transfer lines and the intermachine injection and ejection systems.

The need for integrating the rf equipment into the new PS control system and operating it from the main control room was obvious since the start of the control conversion project in 1978. However, the years between 1978 and 1983 were also the years of the construction of the proton/anitproton collider and the radio-frequency systems were evolving so rapidly that it was extremely difficult to freeze any controls design. Integration of the radio-frequency controls was therefore postponed several times.

In 1984, the different radio-frequency schemes for the collider were more or less frozen and the additions required for LEP were still in the future. Thus a detailed design of both hardware and software was approved in Summer 1984 and the installation of the controls for the first rf subsystems was done during two short shutdowns later in the year. The bulk of the system was installed during the normal longer shutdown early in 1985. In March of the same year the computer control of the radio-frequency systems was operational.

\section{The evolution of the rf systems}

In each accelerator radio-frequency system there are three main entities: the rf cavities, the high-power of generators and their power supplies, and the low-level drive system with facilities of modulation of frequency and voltage applied to the cavities. This modulation is necessary to follow the acceleration cycle and to permit different types of operations.

The PS accelerator is equipped with eleven ferrite loaded cavitities: ten are used in normal operation, the eleventh cavity being a spare. A maximum accelerating voltage of $20 \mathrm{kV}$ per cavity can be attained over a frequency range of 2.6 to $10 \mathrm{MHz}$.

For a long time (1959 to 1976), the operation of the PS consisted essentially of accelerating protons up to high energies for fixed-target physics. Consequently, the rf system was operated in a comparatively simple way: a few kinds of if voltage and frequency programs were sufficient to realize the required acceleration cycles.

The first important increase in complexity came when the PS had to be used as the proton injector for the SPS (1976). A set of eight fixed-tune cavities was installed. These are tuned to the SPS injection frequency, just under $200 \mathrm{MHz}$, and can provide a peak voltage of about $30 \mathrm{kV}$. They are necessary to provide the SPS with properly bunched beam (bunch into bucket transfer) and they permit a clean control of the longitudinal density of particles. These operations, often called "beam gymnastics", required the development of new hardware, mainly for the low-level drive system.

The next increase in complexity came with the antiproton project. The PS accelerator has not only to produce the antiprotons for the AA machine, but it also has to accelerate the antiprotons coming from the AA and send them to the SPS collider. All these new activities required the invention of new gymnastics. These include longitudinal merging [4], special bunch 


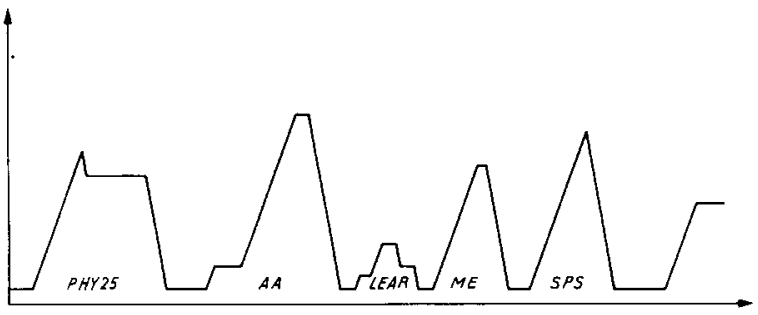

Fig. 1. Typical PS cycles.

rotation and bunch compression [5]. It should be pointed out that all the operations with antiprotons must be carefully designed and executed with a very high reliabiity since a fault occurring during an antiproton bunch transfer means several hours of accumulation lost.

An added complication is that the PS complex is a multi-user proccess. Each one of the machine cycles (of 1-2 s) can be different and dedicated to a specific operation of a particular user. A complete sequence of different cycles is called a supercycle. In the example of fig. 1, the first cycle is used for the physics at $25 \mathrm{GeV}$, the second produces antiprotons for the AA, during the third cycle antiprotons are decelerated to feed the LEAR ring and the fourth cycle is used for machine studies.

In the dead time between two subsequent cycles, all the setting values of the different systems must be changed according to the requirements of the next cycle (pulse-to-pulse modulation). In the case of the rf system, several hundreds of parameters are involved.

\section{The PS control system}

The computer control of the $\mathrm{rf}$ has been realized in the context of the new PS controls system [3]. This system permits a complete control of the PS complex from a central control room using eight general purpose consoles. Each accelerator and each important process has a dedicated front-end computer (FEC), which is a NORD-100 from Norsk Data.

FECs communicate with each other and with the console computers, using a star network with a Nord-10 message handling computer (MHC) (fig. 2). Each FEC has one or more serial CAMAC loops as process interface. A CAMAC crate contains a certain number of interface modules: analog to digital converters, $\mathrm{I} / \mathrm{O}$ registers, timing modules, etc. A microprocessor-based autonomous crate controller (ACC) [6] is present in almost every crate, and is used mainly for two tasks: the local treatment of measurements and the realization of the pulse-to-pulse modulation (PPM).

A specialized computer in the network, the program line sequencer (PLS), generates at the start of each machine cycle information concerning the characteristics of the coming cycle: this information is distributed to each CAMAC crate. Using this information, the

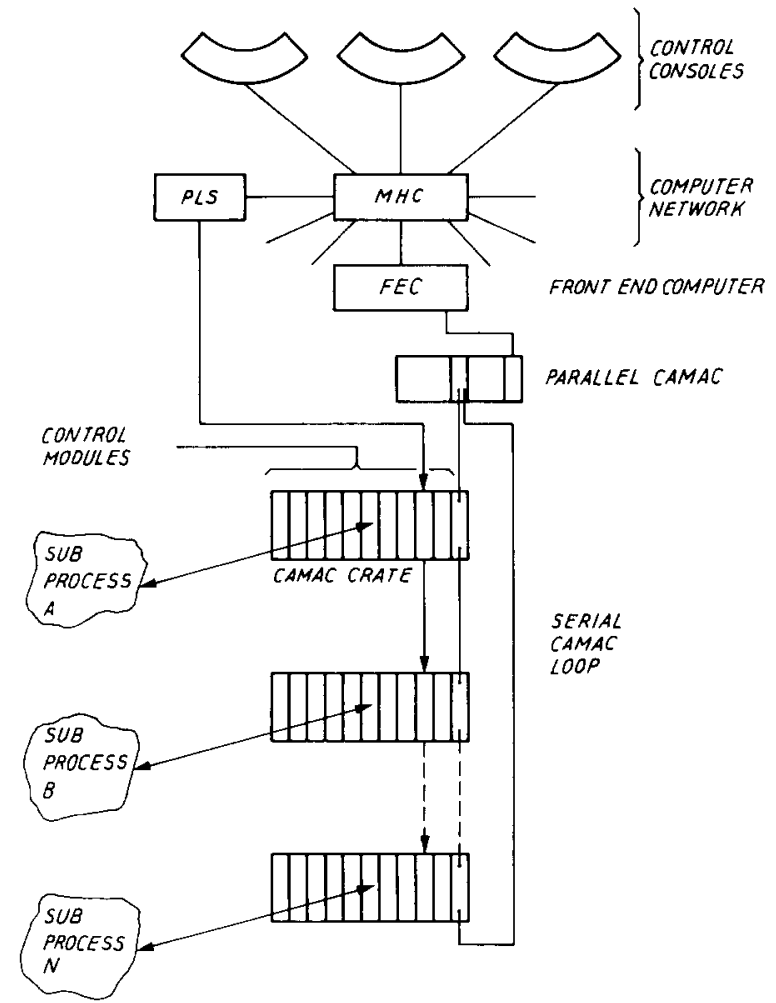

Fig. 2. Simplified PS control layout.

ACC can select for the different control elements the appropriate setting values amongst the ones which are stored in its memory.

The control software is structured in layers according to the hardware:

- The ACC deals with the real-time tasks.

- The FEC computers contain software modules called equipment modules (EM): their main goal is to absorb all the intricacies of the hardware and to present to the user programs a simple and standard calling sequence.

- The console computer contains the sophisticated application programs which use the interactive and display facilities of the consoles.

This general structure has also been applied to the control of the rf systems. The control hardware has been attached to the PS FEC and it occupies a dedicated CAMAC loop with 14 well-filled crates.

\section{The design of the controls of the if systems}

\subsection{The layout team and specufication handbook}

At the beginning of the project, a layout team was created; it was composed of specialists in three different fields: rf systems, controls and operation.

While the role of the first two was to identify the 
requirements and to define how to control the different rf systems, the third one was more specifically interested in how to interact with the controls. Standardization and uniformity had been introduced in the interactive programs from the beginning of the PS controls project.

Specialists analyzed together several aspects of the project:

- Identification of the different systems to be controlled by the computer.

- General design of an adequate control procedure for each system. Where possible, the adopted solution was one which used control tools already well experimented in other systems. In other cases, new hardware and software had to be invented.

- Since the system had to be installed in a number of short shutdowns, the implementation in slices had to be planned. A slice should be composed of systems that functionally and operationally could be converted at the same time.

At the end of this work, about 40 different rf subsystems were identifed (table 1), and the next stage was the production of a specification handbook [7].

This had three main goals:
(1) To freeze the project.

In a changing environment such as the PS complex, it is very important that the different parties agree at a certain moment on a given control philosophy. With the time constraints imposed on the project, this document avoids all the time-consuming discussions on possible modifications during the implementation.

(2) To be used as installation manual.

The information contained in the document is sufficiently detailed to form a clear and unambiguous installation manual for the different control elements, and to be used later for maintenance purposes.

(3) To define the boundary between standard control interface and specific interface. The specification handbook covers that part of the control system called "standard control": this covers the area from the computer down to and including the interface modules contained in the CAMAC crate.

To meet the goals, the specification handbook contains detailed information on the control of each of system. This includes:

- A summary description of the aim and the physics of the subprocess itself. It also exaplains how and in which circumstances the system is used.

Table 1

PS radio frequency systems

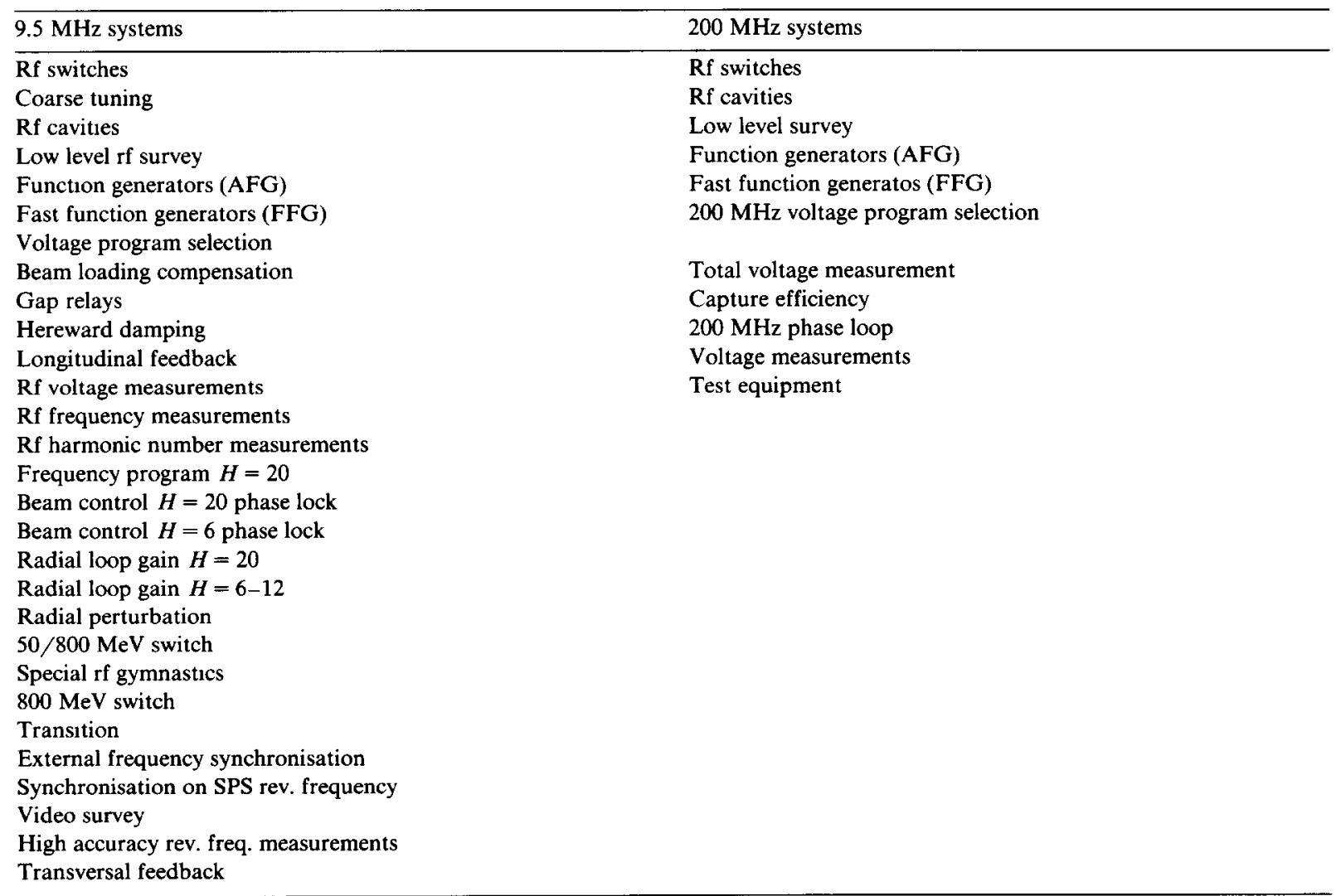


- A list of the parameters to be controlled.

- The hardware control layout. This includes the CAMAC crate and module design and the configuration of each control word with a bit-map.

- The basic software used. This concerns mainly the real-time tasks in the ACC and the equipment modules in the FEC. These basic modules, which are principally hardware oriented, permit any kind of simple control and test on the system.

The specification handbook is completed by a series of tables and diagrams giving information on the general timing pulses distribution, on the interconnections between the standard and the specific interface, etc.

The specification for the more sophisticated and interactive application programs were produced by operation specialists and are described in a separate document [8].

\subsection{Design criteria}

Amongst the $41 \mathrm{rf}$ subsystems, there are differences in size and in complexity. The simplest of them requires the control of only one parameter, while the most complex involves several tens of parameters of different kinds. A subsystem is a subprocess that functionally and/or operationally can be controlled in a independent way.

The advantages of such a subdivision is that the hardware and software control modules can be developed separately, and it eases the problems of implementing the project in separate slices.

Wherever possible, the subsystems were designed so that a system has all its modules in one CAMAC crate and if two or more systems have to share the same CAMAC crate, they should be implemented in the same slice.

Particular care was devoted to reduce the maintenance problems and to provide flexibility for future expansion. The dimension and the complexity of the controls system grow constantly, but the human resources for maintenance cannot follow, for budgetary reasons.

One way to aid maintenance is to separate the different functions. In several cases, we preferred to add an extra CAMAC module, instead of sharing a single module between two functions.

For future expansion, room has been left in the CAMAC crates and the basic software is easily expandable for additional modules of the already existing types.

Machine studies often require the installation and test of new equipment or a different configuration of that existing. For this purpose, a number of standard modules have been installed: all the modules are ready for use with all software and application programs up to the console level. In normal operation, they are not connected to the process. If required for special tests, the connection is made and then the control is immediately active.

Another important point is that the hardware and software were integrated together ab initio in the design of the rf control system. In the past, hardware specialists used to present a "well optimised" hardware design and to ask the software specialists to write some programs "around it". This often produced complicated, expensive and unreliable software. In the case of the if controls, the hardware and the software solution for each system were studied at the same time and the design produced was an optimisation of the two considered as a whole. In many cases, a few modifications in the hardware could save a lot of programming and vice versa.

\subsection{Pulse-to-pulse modulation}

Most of the rf systems have to be modulated from pulse to pulse (PPM). Only the high level systems (cavities) have a static control due to the high power involved.

The PPM system implemented for the other PS processes was not entirely adequate for the if operations and some special arrangement had to be provided. At the start of each PS cycle, the PLS computer sends to the ACC a burst of 128 bits, each one of them representing a condition present in the coming cycle. As already explained, the ACC analyses these bits and selects the setting values for each controlled element from stored tables.

To avoid the complication and large size of the tables if each bit was settable independently, the 128 bits are divided in sets of 8 bits (called groups). Each of the groups represents a characteristic of the cycle: users group, harmonic number group, kind of particles group, etc. Each control element is assigned to a group, so that it needs only eight different setting values at a maximum.

The timing pulses for rf gymnastics do not fit in this philosophy: in many cases a setting value depends, at the same time, on two different groups, e.g. the users and the harmonic number. The solution adopted uses two levels of timing: a main pulse called Master and, if necessary, several cascaded delays per system or per type of gymnastics. By assigning the Master to the group harmonic number and the delays to the group user, one can obtain almost all the required combinations.

Another advantage of this arrangement is that one can displace in time (or just inhibit) a complete operation by acting only on the Master timing.

In total, 36 master timings have been implemented with a variable number of delays. 


\section{The control modules}

About 18 different types of CAMAC modules are used in the rf control system. Three of them are of special importance:

(a) General purpose preset counter (GPPC). This covers a large range of applications. It uses internal clocks or external pulse trains in the range from $1 \mathrm{kHz}$ to $20 \mathrm{MHz}$, to deliver two separate output pulses after counting predefined numbers of clock pulses. Another type of external clock can be used, derived from the magnetic field: : this permits to start an operation at a precise Gauss value. The length and the occurrence of the output pulses can be set to a number of predefined conditions.

(b) Analog function generator ( $A F G$ ). This produces a voltage signal composed of a maximum of 128 vectors. Each vector is adjustable in slope, amplitude and length. The start and stop of the function are realised by external timing pulses. The maximum raising speed is about $1 \mathrm{~V} / 400 \mu \mathrm{s}$.

(c) Fast function generator (FFG). For particular rf operations the maximum slope permitted by the AFG is not sufficient. A fast function generator has therefore been designed specifically for the rf systems. It delivers a two-part function defined in its internal memory (EEPROM) which makes it very versatile, able to generate trapezoids, sinusoids, exponentials, etc.

Two separate clock control words define the time basis of the two parts. The amplitude work is common to both parts, and works like a multiplying coefficient. The maximum slope rate tops at $10 \mathrm{~V} / 32 \mu \mathrm{s}$.

\section{Using the if control system}

As already mentioned, the PS complex is controlled from a main control room using any of the general purpose consoles. For each process of the complex, a certain number of of application programs have been developed and organised into a tree structure. A single program can then be accessed using a touch screen.

For the rf systems, two different trees have been developed: one used for normal operation and the second used by the hardware specialists. Operation and system specialists have different approaches and needs:

- Operators are interested in a structure where the parameters are grouped per kind of operation; the hardware specialist prefers a grouping per hardware system.

- Operators require the control of only a selection of parameters useful for the tuning of different operations; the system specialist needs a complete and detailed control of all the parameters.

- The presentations are also different; displays are more general and condensed for the operators, specific and detailed for the systems specialist.

Amongst the applications programs, those programs that access a large number of parameters are of particular interest.

Three kinds of global operation have been developed:

1) The settings. After a shutdown or after a general crash, these programs permit an automatic initialization of the hardware and the complete restoring of the operational conditions.

2) Operation comparison. The tuning operation of an accelerator usually requires the fine adjustment of several parameters: at a certain moment, the operator wants to check the global effects of his actions on the beam characteristics (intensity, losses, etc.). Using the operation comparison programs, he can immediately restore on the machine the conditions existing at the start of his manipulation and then decide about their efficacity.

3) Archiving. Specific settings of parameters, corresponding to particular operational conditions, can be saved (archived), and then used at a later data by setting programs to restore similar conditions.

\section{Diagnostics}

The maintenance problems and the fault diagnostics have received particular care in the PS control system: the tools mainly used are specific programs and a general alarm system. This latter executes a periodical survey of the different systems and displays exceptions on specialised colour television screens (one for each console). To help in the diagnosis of the rf system, two special tools have been added: a timing surveillance and a cavities diagnostic.

\subsection{Timing survellance}

Rf systems use about 160 timing pulses: their occurrence in precise instants and their relative positioning for a given operation must be rigorously constant. These two conditions are difficult to verify with conventional diagnostic tools. The developed surveillance system uses a logic analyser. All the timing pulses, each with an 8-bit coded name, are connected to the input of the device. Two operational modes are possible:

- in a "one shot" mode it is possible to do a detailed timing analysis on all the pulses occurring during a single cycle (table with pulse names + time of arrival);

- in "surveillance" mode, continuous monitoring highlights pulse sequences which were not identical to the reference ones. Only the order of occurrence of the pulses is analysed in this mode, and not their absolute time of arrival. 


\subsection{Cavities diagnostics}

This system permits to keep track (post mortem) of intermittent and spurious faults in the cavities and coarse tuning hardware. These kinds of faults are very important for the rf specialist but cannot be revealed with the alarm system because the latter presents only a picture of the situation at the moment of the scan. The system is integrated into the same crate containing the control modules of the high level rf systems and is represented in fig. 3 . It is composed of:

- A series of single transceiver modules (a CERN made equipment oriented $\mathrm{I} / \mathrm{O}$ register) controlling the cavities and the coarse tuning system. All the status bits are also required by these modules.

- A counter module. This double module counts the pulses of an external clock ( $1 \mathrm{kHz}$ in our case). The counting is stopped by an external pulse.

- An $\mathrm{I} / \mathrm{O}$ register.

- A clock module which supplies the time and date.

- An ACC module containing a microprocessor with $16 \mathrm{~K}$ of RAM memory.

- A module called program line sequencer receiver (PLSR) which receives all information on the present acclerating cycle from the PLS computer.

The occurrence of a fault or a warning (a less $1 \mathrm{~m}$ portant kind of a fault) produces a pulse signal that stops one or the other channel of the counter: at the same time an interrupt is sent to the microprocessor. This reads:

- from the single transceiver, the complete status of the cavities and of the coarse tuning;

- from the counter, the instant in the cycle in which the fault occurred;

- from the clock module, the time and date;

- from the PLSR module, the particle type, harmonic number, cycle user and number of the supercycle.

In the case of a warning, the number of the cavity that produced it is read in the $\mathrm{I} / \mathrm{O}$ register.

All this information is packed and stored in the memory of the microprocessor. The data of the last 24 faults are always kept in memory. They can be displayed by using appropriate programs.

\section{The measurements}

The correct tuning of the rf system depends on a series of precise measurements. A dedicated microprocessor and a series of appropriate timing modules make these measurements [9].

The most important measurements are:

(1) Gap voltages. These are digitized by an ADC and the results displayed.

(2) $R f$ frequencies. A computer-controlled multiplexer connects the cavity frequency signals to a univer-

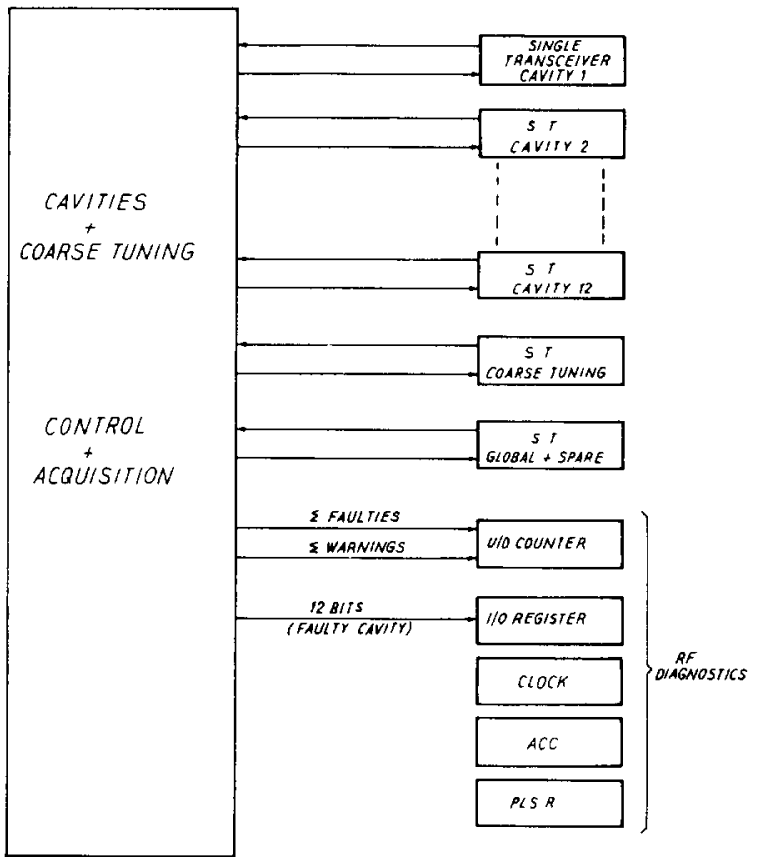

Fig. 3. Cavities diagnostics.

sal counter (HP 5335) to measure the frequencies. This instrument is read out over an HPIB connection by the microprocessor.

(3) Intercavity phase measurements. The same instrument HP 5335 is used, but this time it is programmed as a time interval counter. Two cavities are connected to the two inputs of the instrument and the measured time interval that occurred between the two zero-voltage crossings corresponds to the relative phasing of the cavities.

(4) Harmonic number. The number of radiofrequency periods that occurred during a fixed number of beam revolution frequency periods are read using a counter module (see sect. 7.2) for radio-frequency counts and the GPPC modules for revolution frequency counts. The ratio between these numbers, calculated for each cavity, gives the harmonic number on which the cavity was working at the moment of the measurements.

(5) Revolution frequency. The signal coming from a pickup station is sent to a time interval counter (HP $5370)$. This very high precision measurement $\left(10^{-6}\right.$ for a $100 \mu \mathrm{s}$ gate time) is essential, especially at the moment of the antiproton transfer to the SPS.

The measurement results are displayed on the consoles in different forms. For example, in figs. 4 and 5 the same gap voltage measurements are presented in different ways.

- In fig. 4, the voltages of the eleven cavities at a single instant are displayed with the relative harmonic number per cavity. 


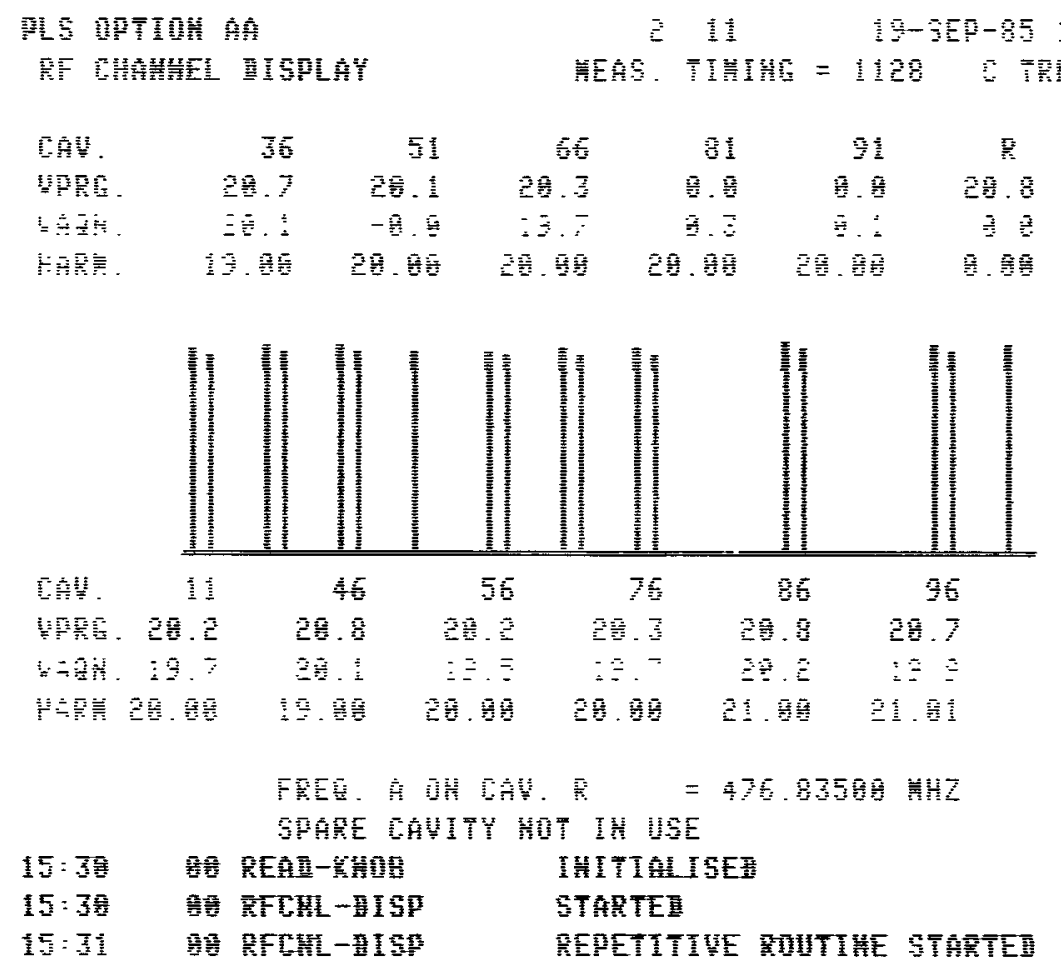

Fig. 4. Gap voltage measurements.

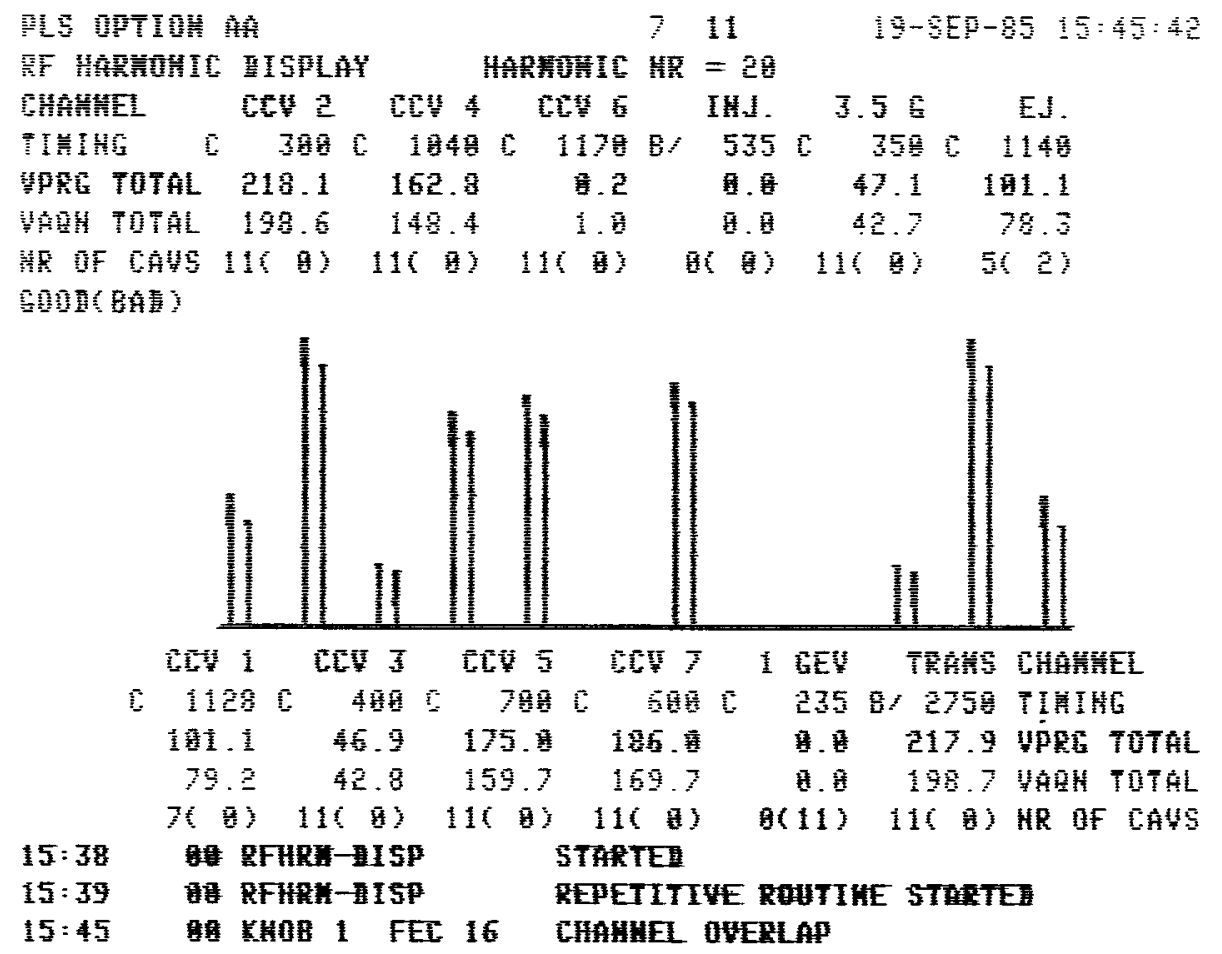

Fig. 5. Measurements on a given harmonic number. 
- In fig. 5, the sums of the voltages have been calculated at different points but only for a given harmonic number (for example on the harmonic 20).

\section{Local control}

Most of the rf systems are grouped in a building located in the centre of the PS ring. A small control console has been installed in this building, with the same characteristics and performance as the consoles in the main control room. This allows the specialist to tune the accelerator and to execute the delicate machine experiments near the specific equipment. At the same time, it has been possible to use the same application software already written and tested for the main consoles.

\section{Resources}

In conclusion we give some figures on the size of the project:

Number of controlled rf systems $\sim 40$

Control hardware modules $\quad \sim 300$

Controlled parameters $\quad \sim 900$

Software development $\sim 9$ man-years
Total PPM throughput (number of words exchanged by micro-processors and standard modules):
control
$\sim 1400$ words $/ \mathrm{s}$
acquisition
$\sim 2000$ words $/ \mathrm{s}$

\section{References}

[1] R. Billinge, Int. Conf. on High Energy Accelerators, Ferm Laboratory, Batavia, Illinois (1983) CERN/PS/83-26 *.

[2] P. Heymans et al., EPS Europhysics Conf. Berln (West) (1983) CERN/PS 83-44 (1983)*.

[3] G. Baribaud et al., IEEE Trans. Nucl. Sci. NS-26 (1979) 3272.

[4] R. Garoby, CERN/PS/LR/Note 80-9 (1980) *.

[5] R. Garoby, CERN(PS/LR/Note 79-16 (1979) *.

[6] F. Beck et al., Esone and European CAMAC Association General Assemblies at DESY, Hamburg (September 1978).

[7] G.P. Benincasa and G. Surback, PS/CO/Note 84-06 (1984) *.

[8] J. Boillot et al., PS/CO/Min. 83-6 (1983) *.

[9] G. Benincasa, F. Giudici and N. Vogt-Nilsen, IFAC, Budapest (July 1984) CERN/PS 84-02 (CO)*.

* CERN Internal Report: available at CERN/PS Secretariat, 1211 Geneva 23, Switzerland. 